\title{
FERMILAB-SLIDES-19-028-T
}

\section{The Global Three-Neutrino Oscillation Picture}

\author{
Iván Martínez Soler
}

Physics Opportunities in the Near DUNE Detector
hall

December 6th, 2018

\section{荤 Fermilab}

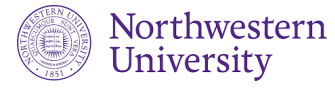


Neutrino evolution

In the $3 \nu$ scenario, neutrino evolution is described by the Schrödinger equation

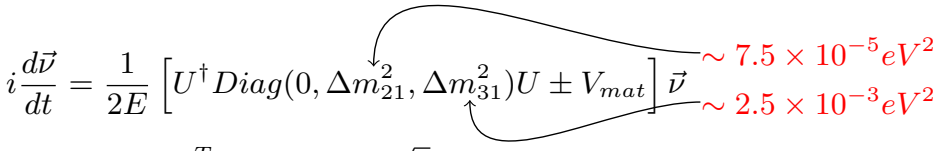

$$
\begin{aligned}
& \vec{\nu}=\left(\nu_{e} \nu_{\mu} \nu_{\tau}\right)^{T} \quad V_{\text {mat }}=\sqrt{2} G_{F} N_{e} \operatorname{Diag}(1,0,0)
\end{aligned}
$$

$U=\left(\begin{array}{ccc}1 & 0 & 0 \\ 0 & c_{23} & s_{23} \\ 0 & -s_{23} & c_{23}\end{array}\right)\left(\begin{array}{ccc}c_{13} & 0 & s_{13} e^{-\delta_{c p}} \\ 0 & 1 & 0 \\ -s_{13} e^{\delta_{c p}} & 0 & c_{13}\end{array}\right)\left(\begin{array}{ccc}c_{12} & s_{12} & 0 \\ -s_{12} & c_{12} & 0 \\ 0 & 0 & 1\end{array}\right) P$

Two possible mass hierarchies
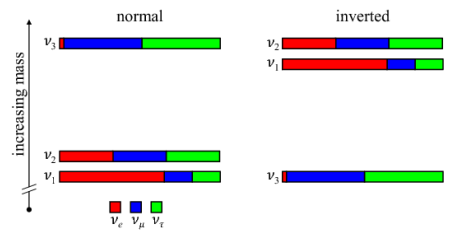

The global fit goal is the determination of the six parameters describing the evolution. 


\section{Neutrino evolution}

In the $3 \nu$ scenario, neutrino evolution is described by the Schrödinger equation

$$
i \frac{d \vec{\nu}}{d t}=\frac{1}{2 E}\left[U^{\dagger} \operatorname{Diag}\left(0, \Delta m_{21}^{2}, \Delta m_{31}^{2}\right) U \pm V_{\text {mat }}\right] \vec{\nu}
$$

$$
U=\left(\begin{array}{ccc}
1 & 0 & 0 \\
0 & c_{23} & s_{23} \\
0 & -s_{23} & c_{23}
\end{array}\right)\left(\begin{array}{ccc}
c_{13} & 0 & s_{13} e^{-\delta_{c p}} \\
0 & 1 & 0 \\
-s_{13} e^{\delta_{c p}} & 0 & c_{13}
\end{array}\right)\left(\begin{array}{ccc}
c_{12} & s_{12} & 0 \\
-s_{12} & c_{12} & 0 \\
0 & 0 & 1
\end{array}\right) P
$$

\begin{tabular}{l|c|c}
\hline Experiment & Dominant & Important \\
\hline Solar & $\theta_{12}$ & $\Delta m_{21}^{2}, \theta_{13}$ \\
Reactor LBL & $\Delta m_{21}^{2}$ & $\theta_{12}, \theta_{13}$ \\
Ractor MBL & $\theta_{13}$ & $\left|\Delta m_{3 l}^{2}\right|$ \\
Atmospheric & $\theta_{23}$ & $\left|\Delta m_{3 l}^{2}\right|, \theta_{13}, \delta_{C P}$ \\
Accelerator LBL $\nu_{\mu}$ Disapp & $\left|\Delta m_{3 l}^{2}\right|, \theta_{23}$ & \\
Accelerator LBL $\nu_{e}$ App & $\delta_{C P}$ & $\theta_{13}, \theta_{23}, \operatorname{sign}\left(\Delta m_{3 l}^{2}\right)$
\end{tabular}


Neutrino evolution

In the $3 \nu$ scenario, neutrino evolution is described by the Schrödinger equation

$$
i \frac{d \vec{\nu}}{d t}=\frac{1}{2 E}\left[U^{\dagger} \operatorname{Diag}\left(0, \Delta m_{21}^{\downarrow_{2}}, \Delta m_{31}^{2}\right) U \pm V_{\text {mat }}\right] \vec{\nu} \sim 7.8 \%
$$

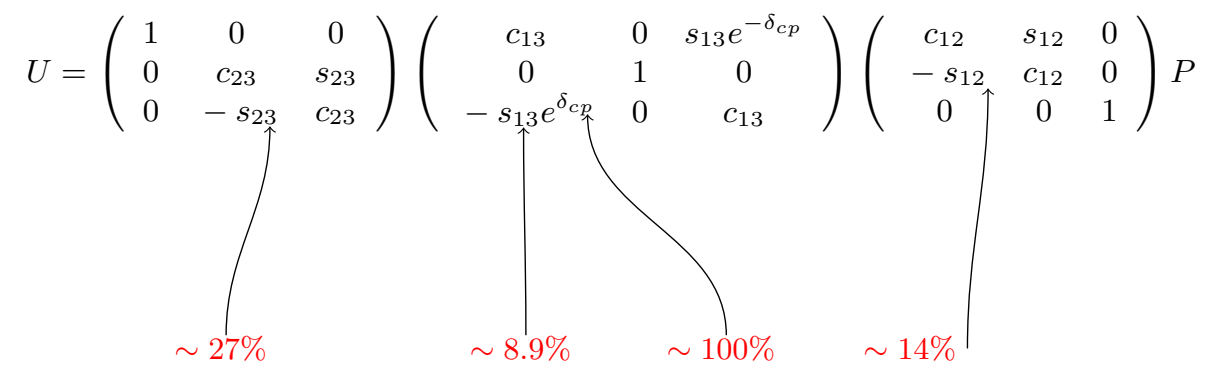


Reactor neutrinos: determination of $\theta_{13}$

- $\bar{\nu}_{e}$ emitted from fission reactions.

- The energy spectrum rise from $1.8 \mathrm{MeV}$ to $4 \mathrm{MeV}$, and falls to very low rate at $8 \mathrm{MeV}$.

- At distances of $\sim 1 \mathrm{~km}[1]$

$$
\begin{aligned}
P_{e e} & =1-c_{13}^{4} \sin ^{2} 2 \theta_{21} \sin ^{2} \Delta_{21}-\sin ^{2} 2 \theta_{13} \sin ^{2} \Delta_{e e} \\
\Delta m_{e e}^{2} & \approx \cos ^{2} \theta_{12}\left|\Delta m_{31}^{2}\right|+\sin ^{2} \theta_{12}\left|\Delta m_{32}^{2}\right|
\end{aligned}
$$

Phys. Rev. D95, 072006 (2017)

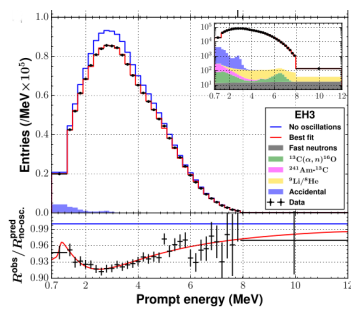

- Reactor neutrinos are sensitive to $\theta_{13}$ and $\Delta m_{31}^{2}$.

- Double-Chooz, RENO and Daya Bay established that $\theta_{13} \neq 0$

- A rate-only analysis determines (Daya Bay Neutrino 2018) $\sin ^{2} 2 \theta_{13}=0.0856 \pm 0.0029$
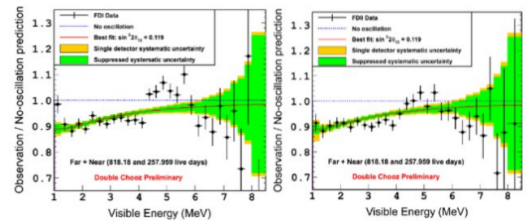

PoS (EPS - HEP2017)109

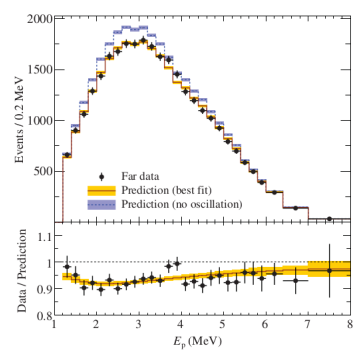

Phys. Rev. D98, 012002 (2018) 


\section{Reactor anomaly}

- The reevaluation of the $\bar{\nu}_{e}$ flux determined a deficit in the experimental data.

- Data/Prediction $=0.952 \pm 0.014 \pm 0.023$ (Daya Bay), $0.918 \pm 0.018$ (RENO)

- A large initial flux prefers larger values of $\theta_{13}$.

- Small impact over $\theta_{13}$ since it is dominated by experiment with near detector.

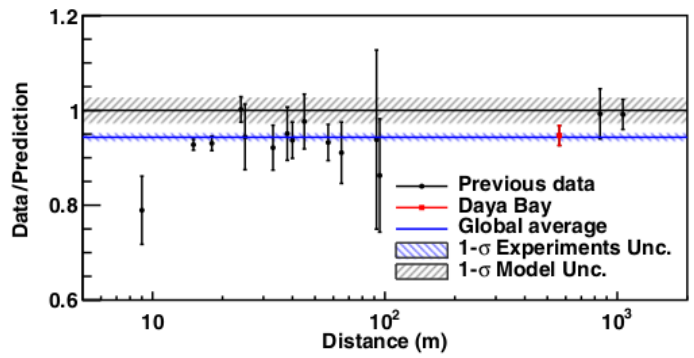

$$
\begin{aligned}
P_{e e} & =1-c_{13}^{4} \sin ^{2} 2 \theta_{21} \sin ^{2} \Delta_{21} \\
& -\sin ^{2} 2 \theta_{13} \sin ^{2} \Delta_{e e}
\end{aligned}
$$


Flux excess at $5 \mathrm{MeV}$

- The ratio of measured over the predicted flux shows an excess at $5 \mathrm{MeV}$.

- The excess is present in all experiments.

- The "bump" is time independent and it is correlated with the reactor power.

- The near/far detector arrangement $\rightarrow \theta_{13}$ depends on the relative uncertainties.
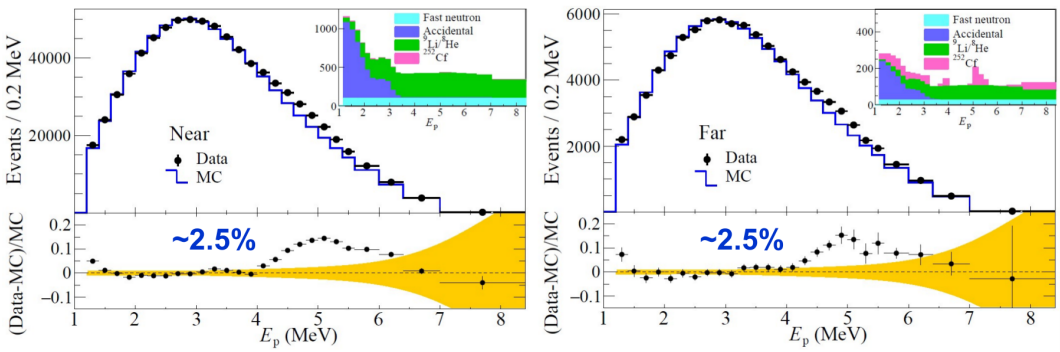

I. Tu (RENO), NEUTRINO 2018 
Reactor neutrinos: determination of $\Delta m_{31}^{2}$

- A spectrum analysis determines $\Delta m_{31}^{2}$

- Near detector imposes an upper bound over $\Delta m_{31}^{2}$.

- The oscillations measured at the far detector impose lower bound on $\theta_{13}$ and $\Delta m_{31}^{2}$

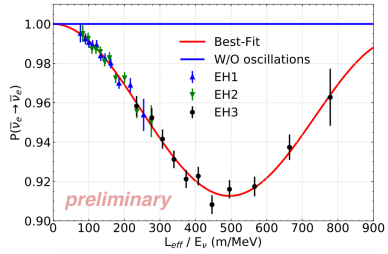

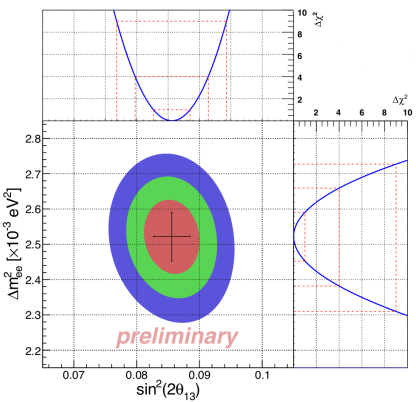

J.P. Ochoa-Recoux (Daya Bay), NEUTRINO 2018
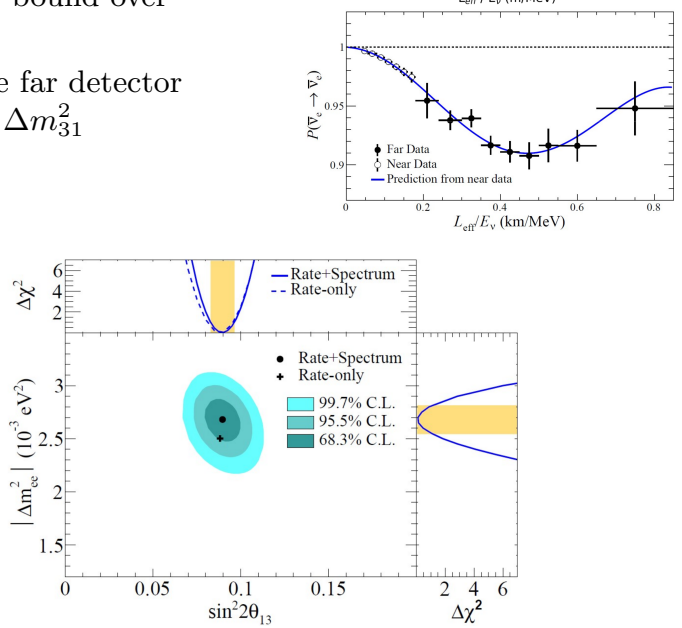

I. Tu (RENO), NEUTRINO 2018 


\section{Solar neutrinos}

- Solar neutrinos are produced by two different nuclear fusion reactions: pp chains and CNO cycles.

$$
4 p \rightarrow{ }^{4} H e+2 e^{+}+2 \nu_{e}+\gamma
$$

- The flux is composed by $\nu_{e}$ with a characteristic energy $\left({ }^{7} B e, p e p\right)$ or spectrum (pp, CNO, ${ }^{8} B$, hep).

Phys. Rev. D 98, 030001 (2018)

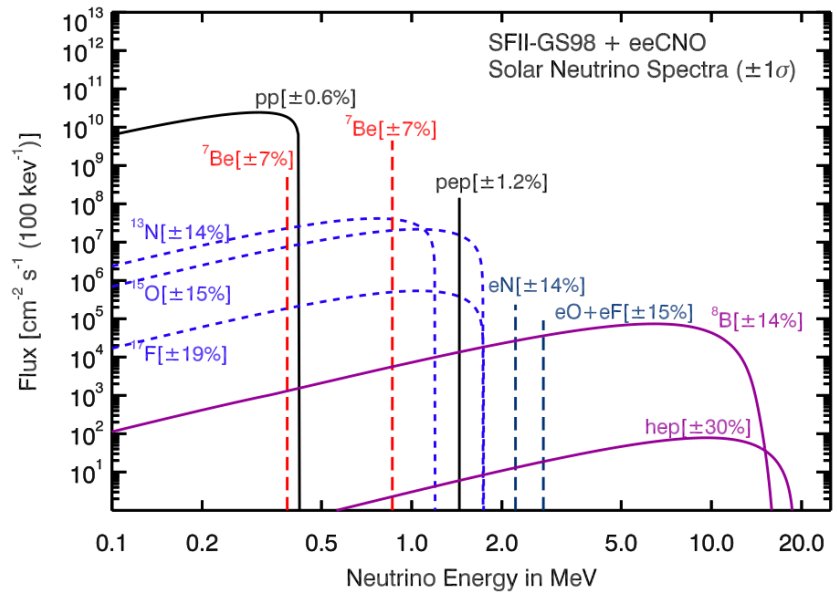


Determination of the solar parameters $\left(\Delta m_{21}^{2}, \theta_{21}\right)$

- $\Delta m_{31}^{2}>>E / L$

$P_{e e}^{3 \nu} \approx \cos ^{2} \theta_{13} \cos ^{2} \theta_{13}^{m} P_{e f f}^{2 \nu}\left(\Delta m_{21}^{2}, \theta_{12}\right)+\sin ^{2} \theta_{13}^{m} \sin ^{2} \theta_{13}$

- For neutrinos created in high densities

$P_{e f f}^{2 \nu}\left(\Delta m_{21}^{2}, \theta_{12}\right)=\frac{1}{2}\left(1+\cos \theta_{12}^{m} \cos \theta_{12}\right)$

- Solar neutrino experiment are mainly sensitive to $\theta_{12}$.

- There is a small dependence on $\Delta m_{21}^{2}$.

- $\theta_{13}^{m}$ carries a slight dependence with $\Delta m_{31}^{2}$.

- The constraints over $\theta_{12}$ are mainly driven by $\mathrm{SK}+\mathrm{SNO}$.

- SNO present a better precision than SK.

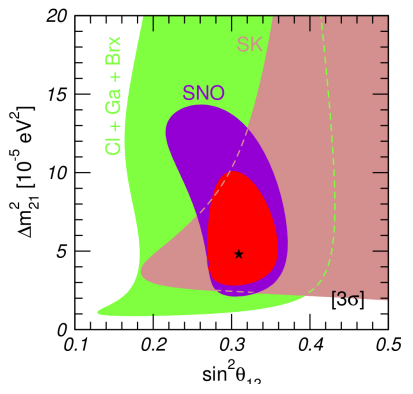

Eur.Phys.J. A52 (2016) no.4, 87

- The results are independent of Solar model used. 
Determination of the solar parameters $\left(\Delta m_{21}^{2}, \theta_{21}\right)$

NuFIT $4.0(2018)$

- $\Delta m_{21}^{2}$ is determined by KamLAND.

- Long-baseline reactor experiment.

- $\bar{\nu}_{e}$ with $E_{\nu} \sim$ few $\mathrm{MeV}$.

- Baseline $\sim 180 \mathrm{~km}$.

$$
\begin{aligned}
& P_{e e}^{3 \nu} \simeq c_{13}^{4} P_{e f f}^{2 \nu}+s_{13}^{4} \\
& \imath \frac{d \vec{\nu}}{d t}=\left[\frac{\Delta m_{21}^{2}}{4 E}\left(\begin{array}{cc}
-\cos 2 \theta_{12} & \sin 2 \theta_{12} \\
\sin 2 \theta_{12} & \cos 2 \theta_{12}
\end{array}\right) \pm \sqrt{2} G_{F} N_{e}\left(\begin{array}{cc}
c_{13}^{2} & 0 \\
0 & 0
\end{array}\right)\right] \vec{\nu}, \vec{\nu}=\left(\begin{array}{c}
\nu_{e} \\
\nu_{a}
\end{array}\right)
\end{aligned}
$$

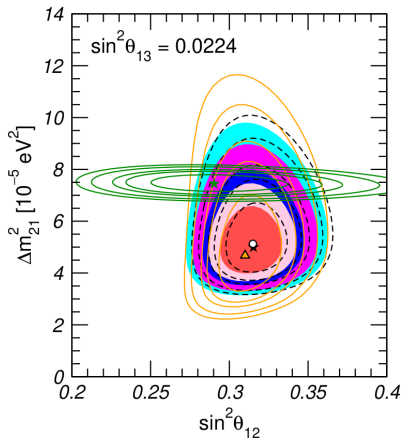

KamLAND determination of $\Delta m_{21}^{2}$ shows a $\sim 2 \sigma$ [1-3] tension with solar 
Tension in $\Delta m_{21}^{2}$

Non observation of low-energy turn up ${ }^{8} B$ neutrino spectrum measured.

Observations indicates:

- $P_{e e} \sim 30 \%$ at high energy ( ${ }^{8} B$, hep).

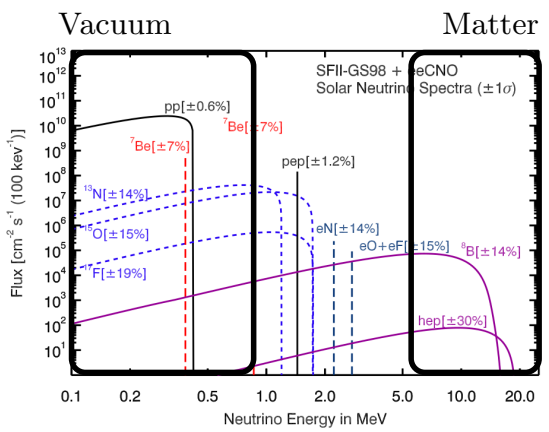

Phys. Rev. D 98, 030001 (2018)
- $P_{e e} \sim 60 \%$ at low energy (pp, ${ }^{7} B e, \mathrm{CNO}$ and low ${ }^{8} B$ ).

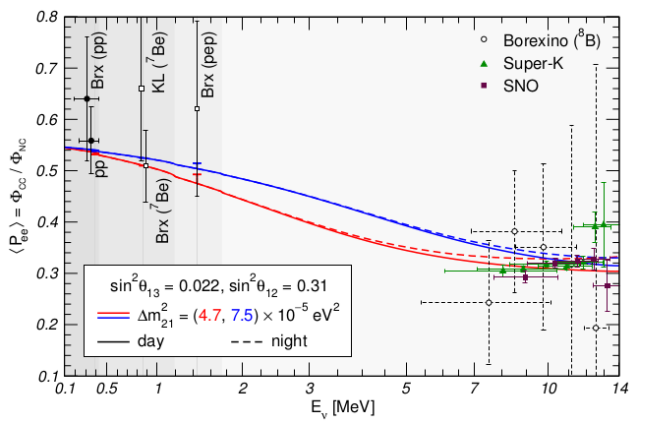

Eur.Phys.J. A52 (2016) no.4, 87 
Tension in $\Delta m_{21}^{2}$

Observation of a larger day/night asymmetry than predicted by KamLAND.
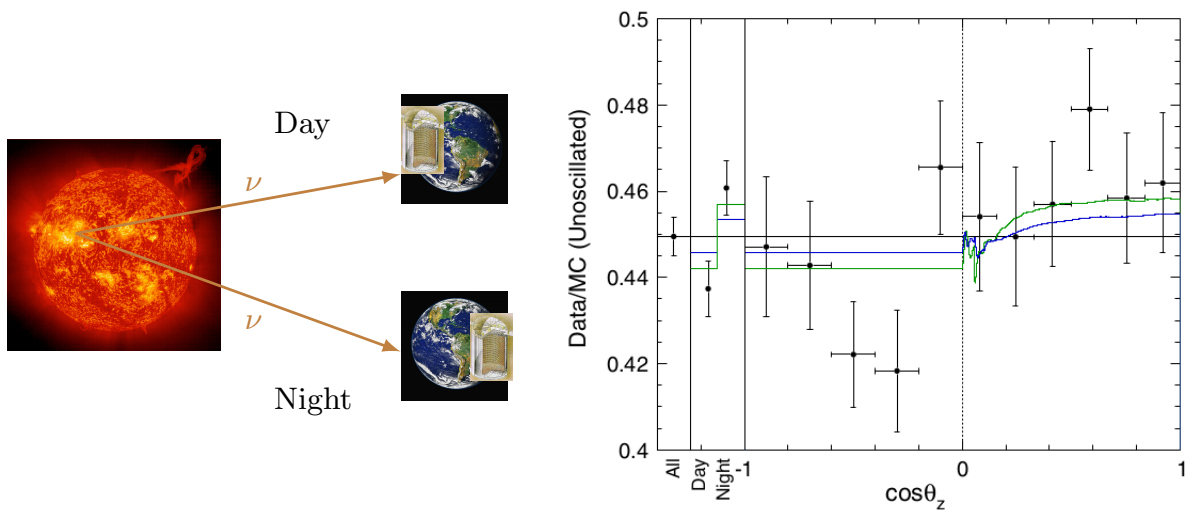

Phys. Rev. D94, 052010 (2016) 
- Created in the collisions of cosmic rays with the atmosphere.

$$
\begin{aligned}
& \pi^{ \pm} \rightarrow \mu^{ \pm}+\nu_{\mu}\left(\overline{\nu_{\mu}}\right) \\
& K^{ \pm} \rightarrow \mu^{ \pm}+\nu_{\mu}\left(\overline{\nu_{\mu}}\right) \\
& \mu^{ \pm} \rightarrow \quad e^{ \pm}+\nu_{e}\left(\overline{\nu_{e}}\right)+\nu_{\mu}\left(\overline{\nu_{\mu}}\right)
\end{aligned}
$$

- Atmospheric flux covers a wide range of energies $\left(\phi_{\nu} \sim E_{\nu}^{-2.7}\right)$.

- The main uncertainties are parametrized by:

- The normalization.

- A energy dependence $\left(\phi_{\nu} \sim \phi_{0}\left(E / E_{0}\right)^{\gamma}\right)$

- The relative contribution of $\pi$ and $\mathrm{K}\left(R_{\pi / K}\right)$

- The ratio between the neutrino and the antineutrino flux $\left(\phi_{\nu} / \phi_{\bar{\nu}}\right)$
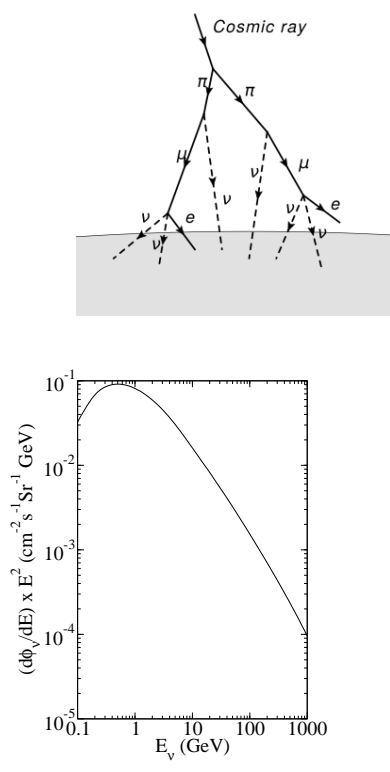

Phys. Rev. D92, 023004 (2015) 
Atmospheric neutrinos: contribution to the global fit

- For $E_{\nu} \geq 0.5 \mathrm{GeV}$ and the earth baselines $\left(10^{2}-10^{4}\right) \mathrm{km} \Delta m_{21}^{2} L / E_{\nu}$ has a subleading effect.

- Atmospheric neutrinos are sensitive to $\Delta m_{31}^{2}, \theta_{23}, \theta_{13}$ and $\delta_{c p}$.

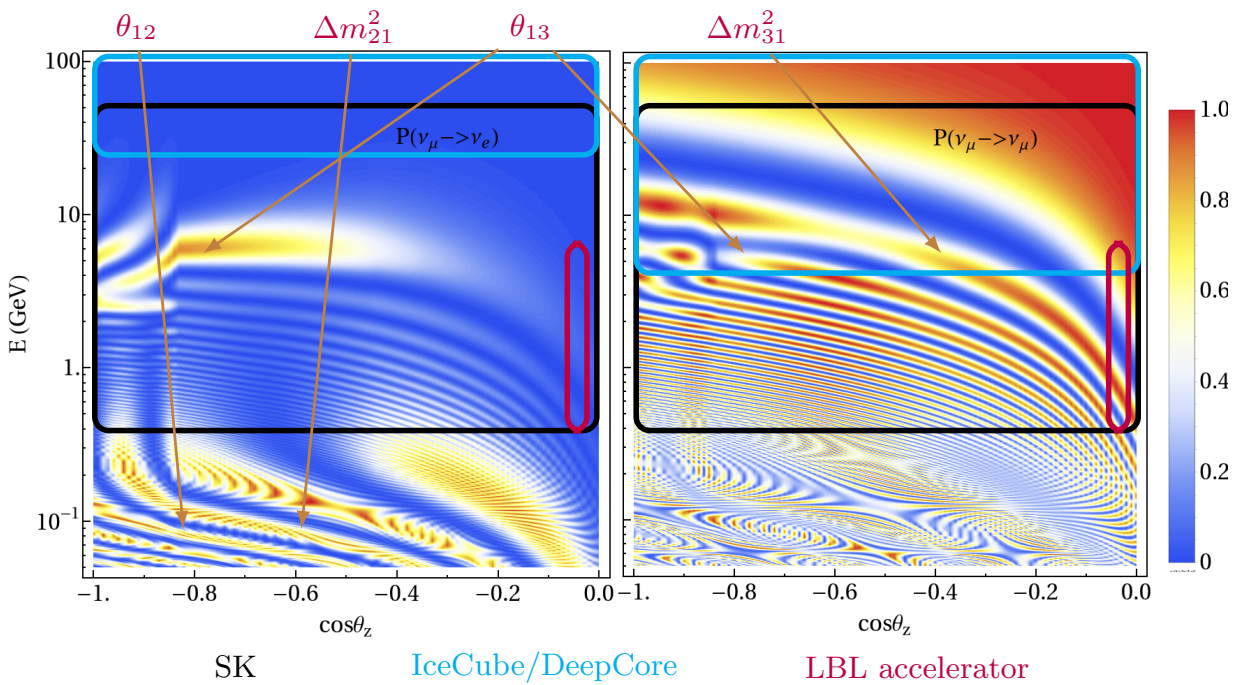


Atmospheric neutrinos: mass hierarchy determination

- 1-3 resonance for $\nu$ crossing the Earth with energies $E_{\nu} \in[2-10] \mathrm{GeV}$.

- For NO (IO) there is a resonance in the $\nu$-channels ( $\bar{\nu}$-channels).

- Atmospheric experiment cannot distinguish $\nu$ from $\bar{\nu}$

- Cherenkov radiation.

- The number of events contains a contribution of $\nu+\bar{\nu}$.

- The neutrino contribution is four times bigger

- Statistical determination of the mass hierarchy.

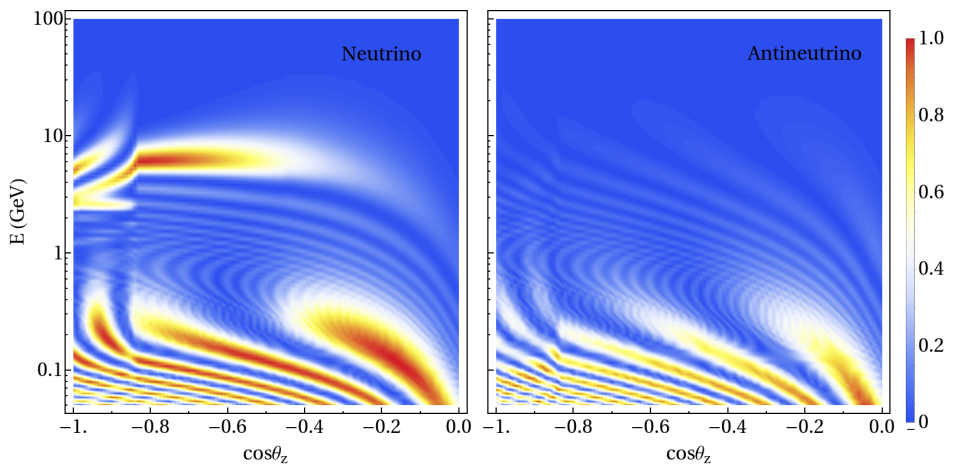


Long-baseline accelerators

- $\nu_{e} / \bar{\nu}_{e}$ and $\nu_{\mu} / \bar{\nu}_{\mu}$ with $E_{\nu} \in[0.6-7] \mathrm{GeV}$

$(\mathrm{T} 2 \mathrm{~K}: \sim 0.6 \mathrm{GeV}, \mathrm{NO} \nu \mathrm{A}: \sim 2 \mathrm{GeV}, \mathrm{MINOS}: \sim 3 \mathrm{GeV}$, MINOS+:

$\sim 7 \mathrm{GeV})$

- The baseline is $\sim 100 \mathrm{~km}$

$(\mathrm{T} 2 \mathrm{~K}: \sim 295 \mathrm{~km}, \mathrm{NO} \nu \mathrm{A}: \sim 810 \mathrm{~km}, \mathrm{MINOS} / \mathrm{MINOS}+: \sim 735 \mathrm{~km})$

- $\nu_{\mu} \rightarrow \nu_{\mu}, \nu_{\mu} \rightarrow \nu_{e}$
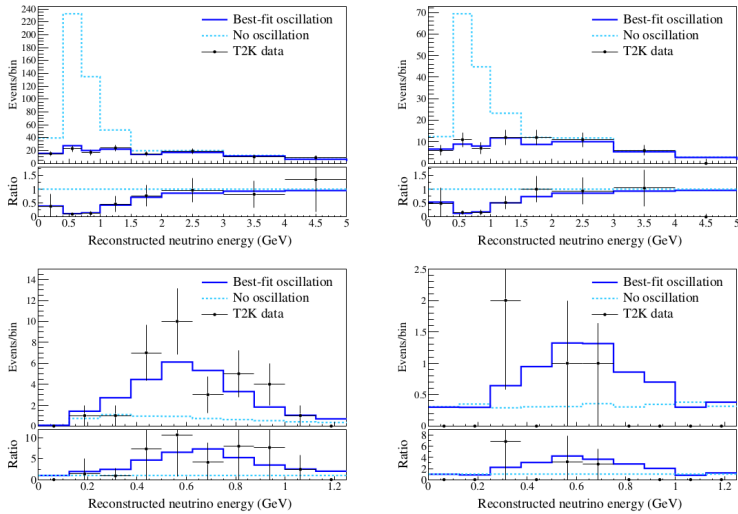

Phys. Rev. D96, 092006 (2017) 
Long-baseline accelerators

- $\nu_{e} / \bar{\nu}_{e}$ and $\nu_{\mu} / \bar{\nu}_{\mu}$ with $E_{\nu} \in[0.6-7] \mathrm{GeV}$

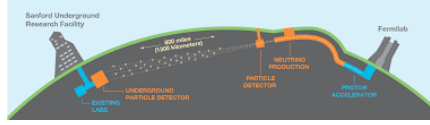
(T2K: $\sim 0.6 \mathrm{GeV}, \mathrm{NO} \nu \mathrm{A}: \sim 2 \mathrm{GeV}$, MINOS: $\sim 3 \mathrm{GeV}$, MINOS+: $\sim 7 \mathrm{GeV}, \mathrm{DUNE}: \sim 2.5 \mathrm{GeV}$ )

- The baseline is $\sim 100 \mathrm{~km}$ (T2K: $295 \mathrm{~km}, \mathrm{NO} \nu \mathrm{A}: \sim 810 \mathrm{~km}$, MINOS/MINOS+: $\sim 735 \mathrm{~km}$, DUNE:1300 km )

- $\nu_{\mu} \rightarrow \nu_{\mu}, \nu_{\mu} \rightarrow \nu_{e}$
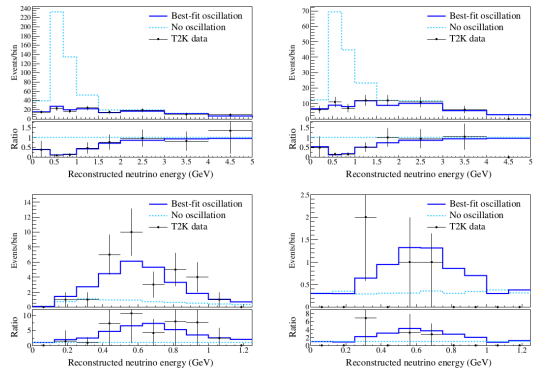
$\nu_{\mu} \rightarrow \nu_{\mu}$ is senstive to $\Delta m_{\mu \mu}^{2}$ and $\theta_{\mu \mu}[1,2]$

$$
P_{\mu \mu} \simeq 1-\sin ^{2} 2 \theta_{\mu \mu} \sin ^{2} \frac{\Delta m_{\mu \mu}^{2} L}{4 E}
$$

where

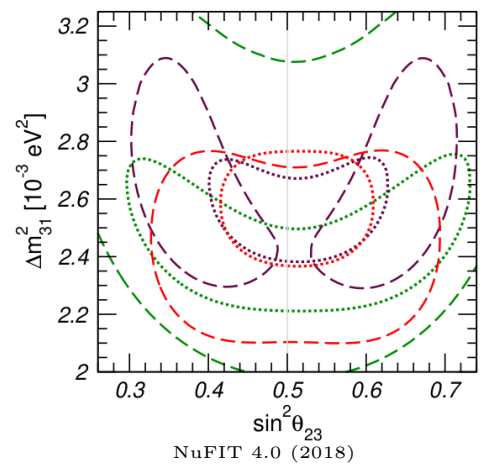

$\Delta m_{\mu \mu}^{2}=\sin ^{2} \theta_{12} \Delta m_{31}^{2}+\cos ^{2} \theta_{12} \Delta m_{32}^{2}+\cos \delta_{c p} \sin \theta_{13} \sin 2 \theta_{12} \tan \theta_{23} \Delta m_{21}^{2}$

- $P_{\mu \mu}$ is symmetric around $\theta_{23} \sim 45$ (maximal mixing or not)

- Discriminate between maximal mixing or not.

- $P_{\mu \mu}$ is sensitive to $\Delta m_{31}^{2}$

[1] Phys. Rev. D72 (2005) 013009

[2] Prog. Theor. Phys. 114 (2006) 1045-1056 
Long-baseline accelerators

$\nu_{e}$ apperance channel

$$
\begin{aligned}
& P_{\nu_{\mu} \rightarrow \nu_{e}} \approx 4 \sin ^{2} \theta_{13} \sin ^{2} \theta_{23}(1+2 o A)-C \sin \delta_{c p}(1+o A) \\
& P_{\overline{\nu_{\mu} \rightarrow \overline{\nu_{e}}}} \approx 4 \sin ^{2} \theta_{13} \sin ^{2} \theta_{23}(1-2 o A)+C \sin \delta_{c p}(1-o A)
\end{aligned}
$$

JHEP 09 (2015) 016

$\nu_{\mu} \rightarrow \nu_{e}$ is sensitive to

- the $\theta_{23}$ octant;

- mass hierarchy;

- $\delta_{c p}$

- $\theta_{13}$.

where

$$
\begin{aligned}
& C=\Delta m_{21}^{2} L / 4 E \sin 2 \theta_{12} \sin 2 \theta_{13} \sin 2 \theta_{23} \\
& o=\operatorname{sign}\left(\Delta m_{31}^{2}\right) \\
& A=\left|2 E V / \Delta m_{31}^{2}\right|
\end{aligned}
$$

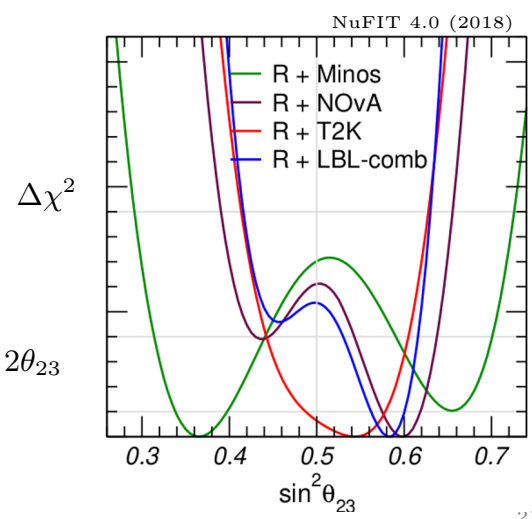


Long-baseline accelerators

$\nu_{e}$ apperance channel

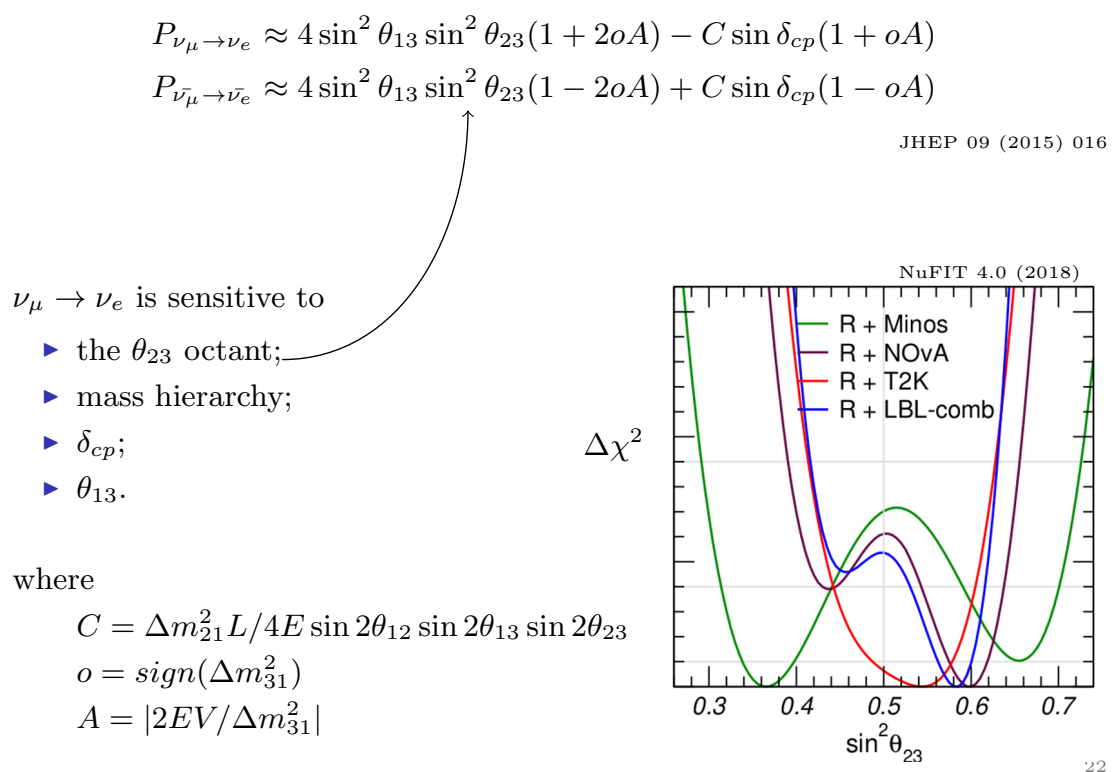


Long-baseline accelerators

$\nu_{e}$ apperance channel

$$
\begin{aligned}
& P_{\nu_{\mu} \rightarrow \nu_{e}} \approx 4 \sin ^{2} \theta_{13} \sin ^{2} \theta_{23}(1+2 o A)-C \sin \delta_{c p}(1+o A) \\
& P_{\overline{\nu_{\mu}} \rightarrow \overline{\nu_{e}}} \approx 4 \sin ^{2} \theta_{13} \sin ^{2} \theta_{23}(1-2 o A)+C \sin \delta_{c p}(1-o A)
\end{aligned}
$$

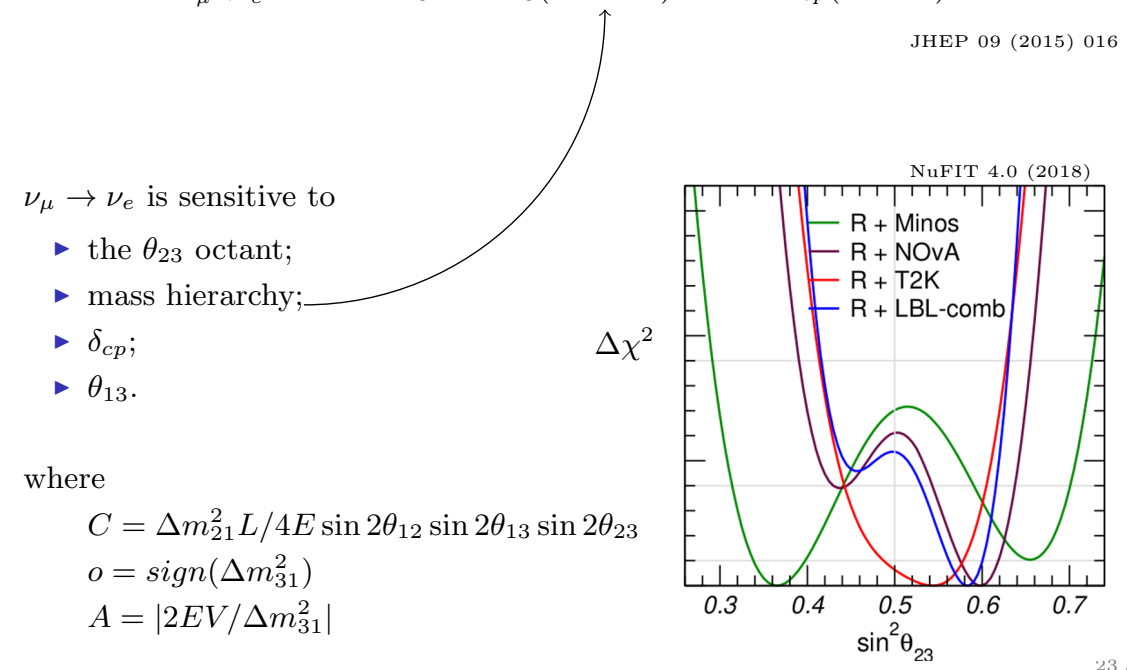

$$
\begin{aligned}
& C=\Delta m_{21}^{2} L / 4 E \sin 2 \theta_{12} \sin 2 \theta_{13} \sin 2 \theta_{23} \\
& o=\operatorname{sign}\left(\Delta m_{31}^{2}\right) \\
& A=\left|2 E V / \Delta m_{31}^{2}\right|
\end{aligned}
$$




\section{Long-baseline accelerators}

$\nu_{e}$ apperance channel

$$
\begin{aligned}
& P_{\nu_{\mu} \rightarrow \nu_{e}} \approx 4 \sin ^{2} \theta_{13} \sin ^{2} \theta_{23}(1+2 o A)-C \sin \delta_{c p}(1+o A) \\
& P_{\overline{\nu_{\mu}} \rightarrow \overline{\nu_{e}}} \approx 4 \sin ^{2} \theta_{13} \sin ^{2} \theta_{23}(1-2 o A)+C \sin \delta_{c p}(1-o A)
\end{aligned}
$$

$\nu_{\mu} \rightarrow \nu_{e}$ is sensitive to

- the $\theta_{23}$ octant;

- mass hierarchy;

- $\delta_{c p}$

- $\theta_{13}$.

where

$$
\begin{aligned}
& C=\Delta m_{21}^{2} L / 4 E \sin 2 \theta_{12} \sin 2 \theta_{13} \sin 2 \theta_{23} \\
& o=\operatorname{sign}\left(\Delta m_{31}^{2}\right) \\
& A=\left|2 E V / \Delta m_{31}^{2}\right|
\end{aligned}
$$

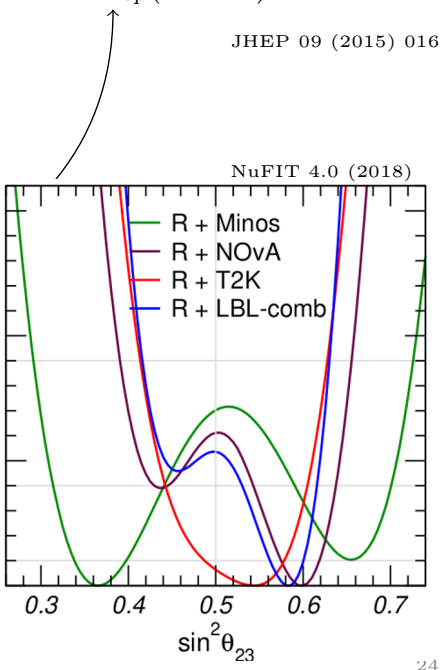




\section{Long-baseline accelerators}

$\nu_{e}$ apperance channel

$$
\begin{aligned}
& P_{\nu_{\mu} \rightarrow \nu_{e}} \approx 4 \sin ^{2} \theta_{13} \sin ^{2} \theta_{23}(1+2 o A)-C \sin \delta_{c p}(1+o A) \\
& P_{\overline{\nu_{\mu}} \rightarrow \overline{\nu_{e}}} \approx 4 \sin ^{2} \theta_{13} \sin ^{2} \theta_{23}(1-2 o A)+C \sin \delta_{c p}(1-o A)
\end{aligned}
$$

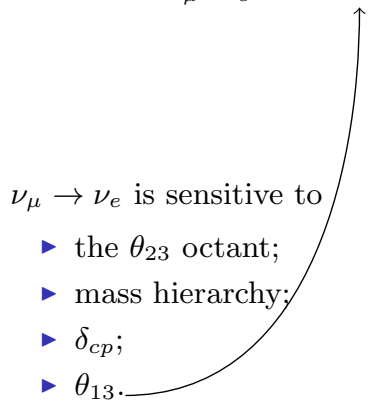

where

$$
\begin{aligned}
& C=\Delta m_{21}^{2} L / 4 E \sin 2 \theta_{12} \sin 2 \theta_{13} \sin 2 \theta_{23} \\
& o=\operatorname{sign}\left(\Delta m_{31}^{2}\right) \\
& A=\left|2 E V / \Delta m_{31}^{2}\right|
\end{aligned}
$$

NuFIT 4.0 (2018)

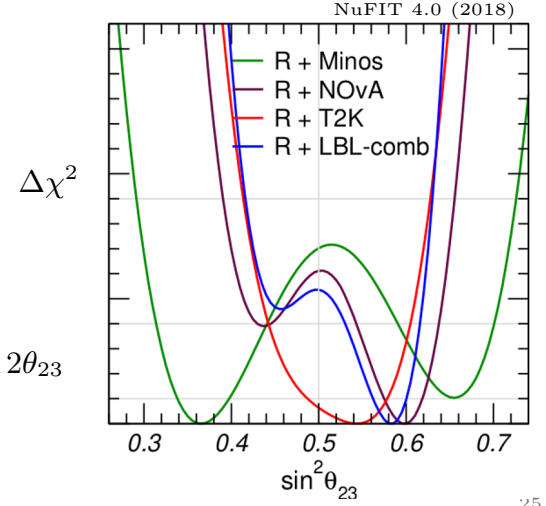


Determination of $\theta_{23}$ and $\Delta m_{31}^{2}$

- Preference for the second octant of $\theta_{23}$

- Maximal mixing is disvafor by:

- T2K (app. channel)

- NO $\nu \mathrm{A}(\bar{\nu}$-disapp. channel and app. channel ) NuFIT $4.0(2018)$

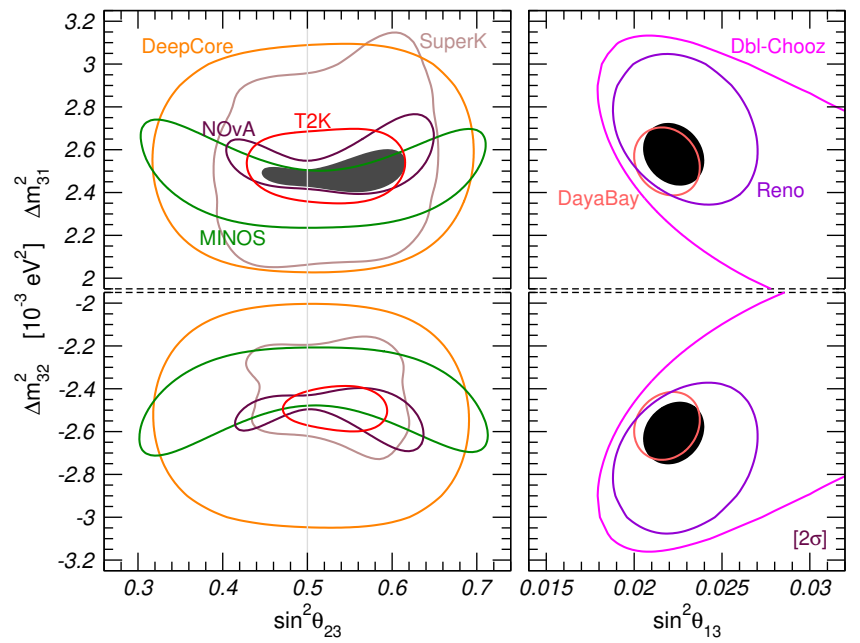


Determination of mass hierarchy

Preference for $\mathrm{NH} \sim 3 \sigma$.

- SK (SK + T2K) (bounds $\theta_{13}$ from reactors): favor $\mathrm{NH} \sim 2.1 \sigma(\sim 2.3 \sigma)$

- T2K (adding $\theta_{13}$ from reactors): $\mathrm{NH}$ favored $\sim 2 \sigma$

- $\mathrm{NO} \nu \mathrm{A}: \mathrm{NH}$ favored $\sim 1.3 \sigma$

- MINOS + MINOS+: very weak preference for $\mathrm{NH} \sim 0.6 \sigma$.

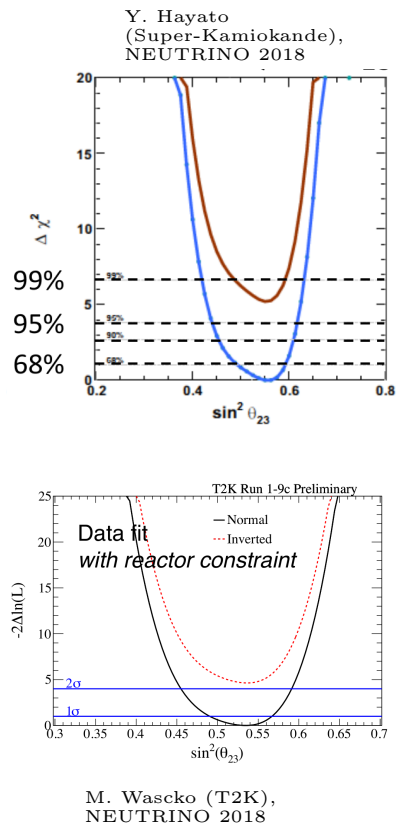

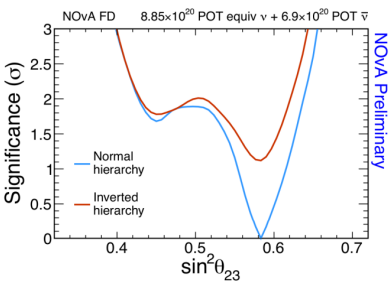

M. Sanchez $(\mathrm{NO} \nu \mathrm{A})$, NEUTRINO 2018

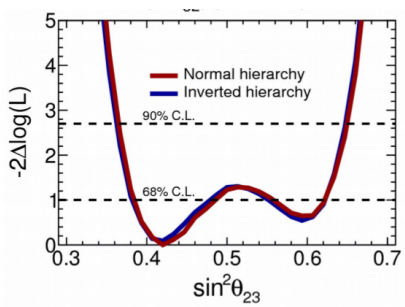

A. Aurisano (MINOS and MINOS+), NEUTRINO 2018 
- The best-fit of LBL shows $\delta_{c p}=215^{\circ}$

- T2K and $\mathrm{NO} \nu \mathrm{A}(\mathrm{IO})$ prefer maximal $\mathrm{CP}$ violation $\left(\delta_{C P} \sim 270^{\circ}\right)$

- $\mathrm{NO} \nu \mathrm{A}(\mathrm{NO})$ prefer $\delta_{C P} \sim 30^{\circ}$

- SK favor $\delta_{c p}=270^{\circ}$
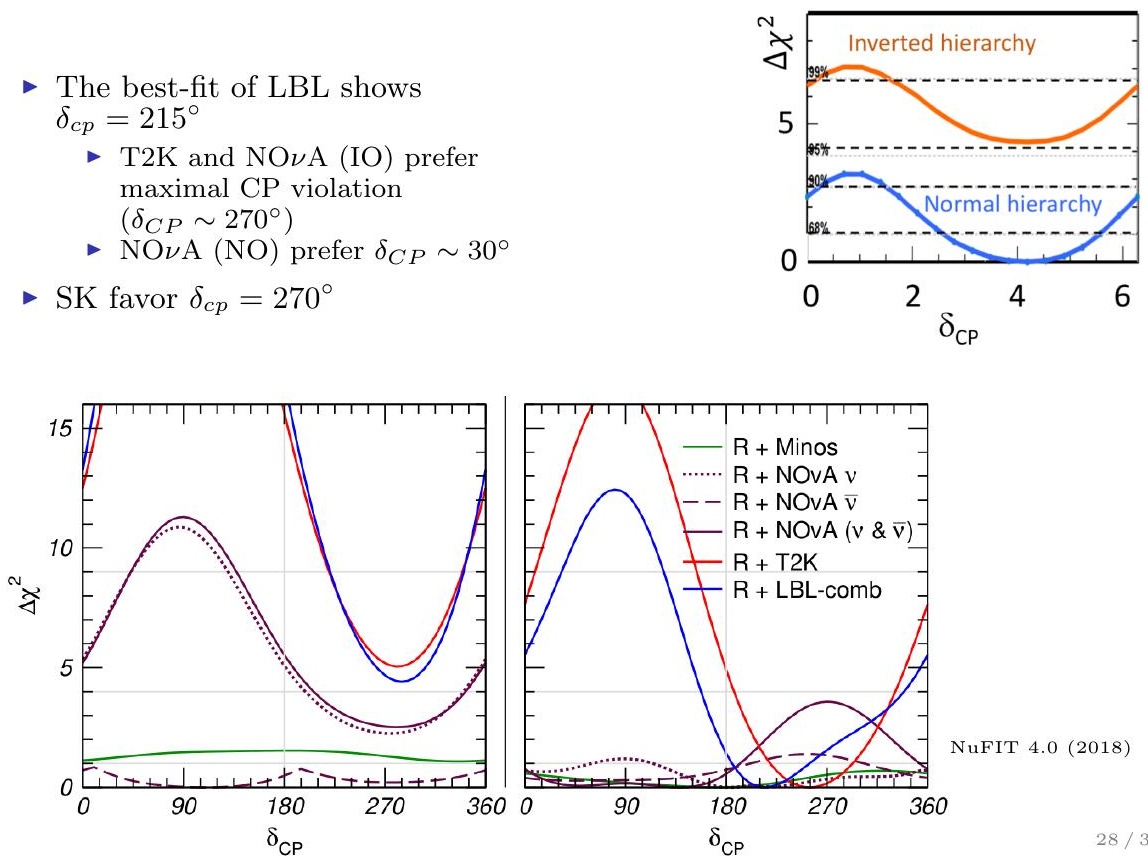


\section{Conclusions}

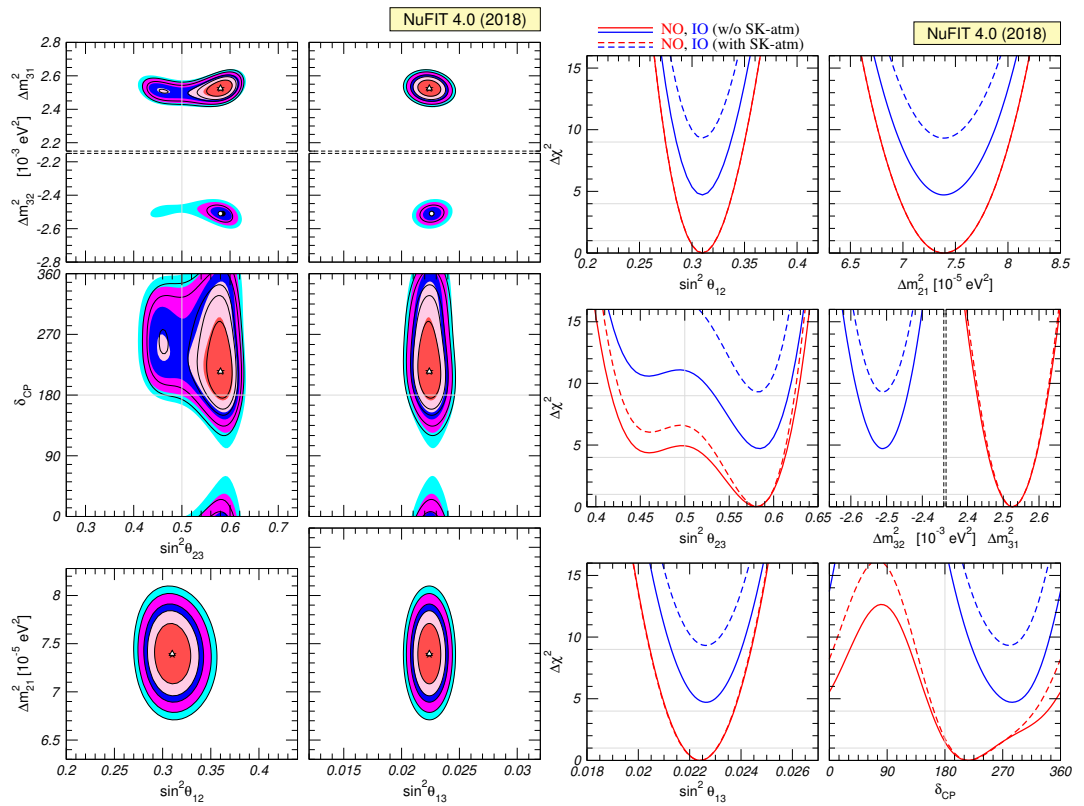




\section{Conclusions}

Comparison between different global fits

\begin{tabular}{|c|c|c|c|}
\hline & Esteban et al., [1] & Capozzi et al.,[2] & Salas et al.,[3] \\
\hline $\sin ^{2} \theta_{12}$ & $0.310_{-0.012}^{+0.013}$ & $0.304_{-0.013}^{+0.014}$ & $0.320_{-0.16}^{+0.20}$ \\
$\sin ^{2} \theta_{23}$ & $0.580_{-0.021}^{+0.017}$ & $0.551_{-0.070}^{+0.019}$ & $0.547_{-0.30}^{+0.20}$ \\
$\sin ^{2} \theta_{13}$ & $0.02241_{-0.00065}^{+0.00065}$ & $0.0214_{-0.0007}^{+0.0009}$ & $0.0216_{-0.00069}^{+0.00083}$ \\
$\delta_{C P}$ & $215_{-29}^{+40}$ & $234_{-32}^{+41}$ & $218_{-27}^{+38}$ \\
$\frac{\Delta m_{21}^{2}}{10^{-5} \mathrm{eV}^{2}}$ & $7.39_{-0.20}^{+0.21}$ & $7.34_{-0.14}^{+0.17}$ & $7.55_{-0.16}^{+0.20}$ \\
$\frac{\Delta m_{31}^{2}}{10^{-3} \mathrm{eV}^{2}}$ & $2.525_{-0.031}^{+0.033}$ & $2.455_{-0.032}^{+0.035}$ & $2.50_{-0.03}^{+0.03}$ \\
\hline
\end{tabular}

[1] I. Esteban, et al., arXiv:1811.05487, NuFIT 4 (2018), www.nu-fit.org

[2] F. Capozzi, E. Lisi, A. Marrone, and A. Palazzo, Prog.Part.Nucl.Phys. 102 (2018) 48-72

[3] P.F. de Salas, D.V. Forero, C.A. Ternes, M. Tortola, J.W.F. Valle, Phys.Lett. B782 (2018) 633-640 
Thank you! 


\section{KamLAND and the $5 \mathrm{MeV}$ excess}

- There is no a near detector in KamLAND.

- the flux can be affected by the excess around $E_{\nu} \sim 5 \mathrm{MeV}$.

- There is a small impact on the determination of $\Delta m_{21}^{2}$
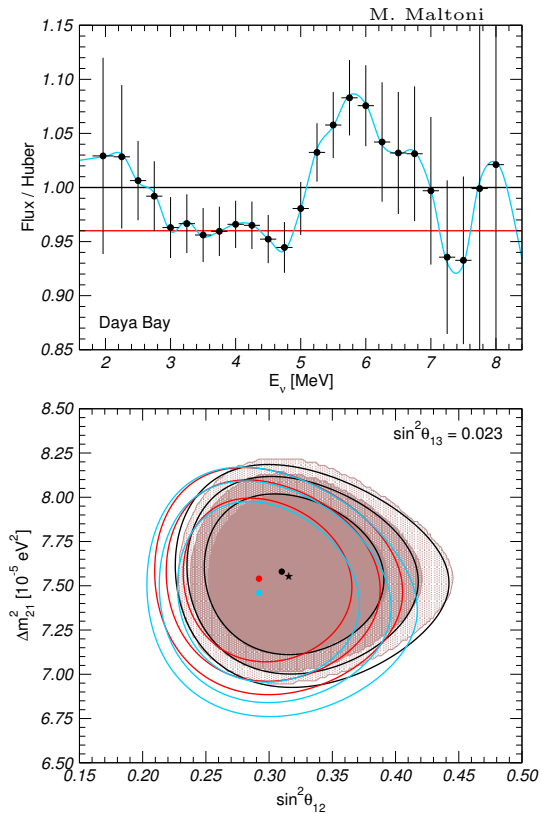